\title{
A DIAGNOSTIC FOR MODELS OF U GEMINORUM
}

B. Paczyriski and A. Schwarzenberg-Czerny

Copernicus Astronomical Center, Warsaw, Poland

and

Charles Alcock

Institute for Advanced Study, Princeton, NJ 08540

Models of $U$ Geminorum fall into two basic types. Both involve a Roche-lobe filling main sequence dwarf orbiting a white dwarf, around which there is an accretion disk. In one type of model there is continuous mass accretion from the inner Lagrange point through the disk to the white dwarf at all times, and the outburst is produced by varying the mass transfer across the inner Lagrange point (Bath et al., 1974). In the second type of model, there is not any accretion from the outer edge of the disk to the white dwarf between outbursts, and matter streaming from the inner lagrange point accumulates in a torus (Osaki 1974, Paczynski 1978). The outburst is a result of an instability in the disk that suddenly increases the viscosity. A simple diagnostic test of the former type of model is to model the luminosity produced from the accretion disk at minimum light. Following Smak (1976) we adopt a white dwarf mass of $0.9 \mathrm{M}_{\odot}$, inner and outer radii of the disk of $6.1 \times 10^{8} \mathrm{~cm}$ and $3.6 \times 10^{10} \mathrm{~cm}$, inclination $67^{\circ}$, and velocity of the stream hitting the disk of $400 \mathrm{~km} \mathrm{sec-1.} \mathrm{Following} \mathrm{Wade} \mathrm{(1979)} \mathrm{we}$ adopt a distance modulus of $4 \mathrm{~m} .4$ and a maximum brightness in the visual band of $14 \mathrm{~m} .76$. Averaging this radiation over angles and using the velocity information gives a lower limit to the mass transfer rate of $10^{-10} \mathrm{M}_{\odot} \mathrm{yr}^{-1}$. Assuming that the disk is optically thick, and taking into account limb darkening in an approximate fashion, yields an expected brightness in the visual band $V_{\text {disk }} \leq 12^{m} \cdot 6$. This is two magnitudes too bright. A crude analysis of optically thin disks indicates that stable configurations only exist for disk temperatures close to $1.5 \times 10^{4} 0_{K}$. This temperature is too low for the accretion rate inferred here, if the disk is optically thin. For this reason the disk must be optically thick. Since the visual magnitude of the disk is too high if it is accreting, we conclude that continuous accretion does not occur at minimum light and that matter accumulates in a torus.

Bath, G. T., Evans, W. D., Papaloizou, J. and Pringle, J. E. 1974 , M.N.R.A.S. 169,447 .

Osaki, Y. 1974, Pub. Astr. Soc. Japan, 26, 429.

Paczyúski, B. 1978, in "Nonstationary Evolution of Close Binaries" ed.

A. N. Zytkow (PWN: Warsaw) p. 89.

Smak, J. 1976 Acta, Astr. 26, 277.

Wade, R. 1979 A. J. 84, 562: 